\title{
Statin Treatment Is Associated with Reduction in Serum Levels of Receptor Activator of NF- $\kappa$ B Ligand and Neutrophil Activation in Patients with Severe Carotid Stenosis
}

\author{
Sébastien Lenglet, ${ }^{1}$ Alessandra Quercioli, ${ }^{1}$ Mathias Fabre, ${ }^{1}$ Katia Galan, ${ }^{1}$ Graziano Pelli, ${ }^{1}$ \\ Alessio Nencioni, ${ }^{2}$ Inga Bauer, ${ }^{2}$ Aldo Pende, ${ }^{3}$ Magaly Python, ${ }^{1}$ Maria Bertolotto, ${ }^{3}$ \\ Giovanni Spinella, ${ }^{4}$ Bianca Pane, ${ }^{4}$ Domenico Palombo, ${ }^{4}$ Franco Dallegri, ${ }^{3}$ François Mach, \\ Nicolas Vuilleumier, ${ }^{5}$ and Fabrizio Montecucco ${ }^{1,3,5}$ \\ ${ }^{1}$ Division of Cardiology, Department of Internal Medicine, Foundation for Medical Researches, University of Geneva, \\ 64 Avenue de la Roseraie, 1211 Geneva, Switzerland \\ ${ }^{2}$ Department of Internal Medicine, University of Genoa, 6 Viale Benedetto XV, 16132 Genoa, Italy \\ ${ }^{3}$ First Clinic of Internal Medicine, Department of Internal Medicine, University of Genoa School of Medicine, \\ IRCCS Azienda Ospedaliera Universitaria San Martino-IST Istituto Nazionale per la Ricerca sul Cancro, Viale Benedetto XV, \\ 16132 Genoa, Italy \\ ${ }^{4}$ Vascular and Endovascular Surgery Unit, Department of Surgery, San Martino Hospital, 10 Largo Rosanna Benzi, 16132 Genoa, Italy \\ ${ }^{5}$ Division of Laboratory Medicine, Department of Genetics and Laboratory Medicine, Geneva University Hospitals, \\ 4 Rue Gabrielle-Perret-Gentil, 1205 Geneva, Switzerland
}

Correspondence should be addressed to Fabrizio Montecucco; fabrizio.montecucco@unige.ch

Received 12 September 2013; Accepted 22 December 2013; Published 6 February 2014

Academic Editor: Massimiliano M. Corsi Romanelli

Copyright (C) 2014 Sébastien Lenglet et al. This is an open access article distributed under the Creative Commons Attribution License, which permits unrestricted use, distribution, and reproduction in any medium, provided the original work is properly cited.

\begin{abstract}
Systemic and intraplaque biomarkers have been widely investigated in clinical cohorts as promising surrogate parameters of cardiovascular vulnerability. In this pilot study, we investigated if systemic and intraplaque levels of calcification biomarkers were affected by treatment with a statin in a cohort of patients with severe carotid stenosis and being asymptomatic for ischemic stroke. Patients on statin therapy had reduced serum osteopontin (OPN), RANKL/osteoprotegerin (OPG) ratio, and MMP-9/pro-MMP9 activity as compared to untreated patients. Statin-treated patients exhibited increased levels of collagen and reduced neutrophil infiltration in downstream portions of carotid plaques as compared to untreated controls. In upstream plaque portions, OPG content was increased in statin-treated patients as compared to controls. Other histological parameters (such as lipid, macrophage, smooth muscle cell, and MMP-9 content) as well as RANKL, RANK, and OPG mRNA levels did not differ between the two patient groups. Serum RANKL/OPG ratio positively correlated with serum levels of neutrophilic products, intraplaque neutrophil, and MMP-9 content within downstream portions of carotid plaques. In conclusion, statin treatment was associated with improvement in serum RANKL levels and reduced neutrophil activity both systemically and in atherosclerotic plaques.
\end{abstract}

\section{Introduction}

Atherosclerotic plaque calcification is considered a common process in advanced atherogenesis, although its association with increased plaque vulnerability remains unclear. On the one hand, the coronary calcium score (CAC) was shown to improve the Framingham risk score prediction in intermediate risk individuals [1] and to be an independent predictor of stroke in subjects deemed at low risk (accordingly to clinically based traditional risk stratifications tools) [2]. On the other hand, histological findings from human carotid atherosclerotic plaques demonstrated that symptomatic atheroma is characterized by reduced levels of calcification and increased inflammation as compared to 
asymptomatic plaques [3]. This scenario is further complicated by recent recommendations that "intraplaque vulnerability" is just considered as one of multiple determinants of cardiovascular risk (i.e., intraplaque, circulating, and peripheral tissue risk factors), suggesting the importance of defining systemic risk biomarkers and of understanding their pathophysiological role $[4,5]$. Potential "systemic" biomarkers of intraplaque calcification include osteopontin (OPN), osteoprotegerin (OPG), and receptor activator of nuclear factor $\mathrm{kB}$ ligand (RANKL) [6-8]. In this context, we have recently shown that RANKL promotes the degranulation liberating proatherosclerotic products by neutrophils [9]. Notably, specific anti-RANKL neutralizing antibodies for clinical applications (i.e., denosumab) have been developed and are currently investigated as a way to block skeletal bone resorption in cancer diseases [10]. Since cardiovascular outcomes were not considered as endpoints in available cancerrelated trials [11], the potential effects of those therapeutic compounds on atherogenesis are still elusive, and knowing whether conventional antiatherosclerotic treatments could affect RANKL signalling remains an open question.

Therefore, in this work, we studied the potential antiRANKL effects of the 3-hydroxy-3-methylglutaryl-coenzyme A (HMG-CoA) reductase inhibitors (statins) in patients with severe carotid plaque stenosis and being asymptomatic for acute ischemic diseases.

Statins are the most common lipid-lowering drugs worldwide and are recommended for the prevention of cardiovascular diseases [12]. Their antiatherosclerotic effects include not only lipid profile improvements, but also additional "pleiotropic" activities (such as anti-inflammatory, antioxidative, and immunomodulatory activities), which all contribute to stabilizing atherosclerotic plaques $[13,14]$ and reduction of systemic atherosclerotic factors, such as C-reactive protein (CRP) [15]. For these reasons, statins might be very promising candidates to potentially reduce systemic and intraplaque RANKL levels and/or bioactivity (as a neutrophil activator) in advanced atherosclerosis.

\section{Methods}

2.1. Patients and Study Design. We conducted an observational cohort study between December 2010 and November 2011 at a single hospital (San Martino Hospital) in Genoa (Italy). Patients $(n=38)$ undergoing carotid endarterectomy (CEA) for extra cranial high-grade internal carotid stenosis (>70\% luminal narrowing) [4-6] and being asymptomatic for ischemic stroke were included in the study. Patients were defined as asymptomatic when they had no history of ischemic symptoms and in the absence of signs of cerebral necrosis at magnetic resonance imaging (MRI) with diffusion sequences. The exclusion criteria were malignant hypertension, acute coronary artery disease, any cardiac arrhythmias, congestive heart failure (II, III, and IV NHYA classes), liver or renal disorders or function abnormalities, acute and chronic infectious disease, autoimmune and rheumatic diseases, osteoporosis, cancer, endocrine diseases, inflammatory bowel diseases and anti-inflammatory (other than aspirin) medications, oral anticoagulant treatments, hormone, and cytokine or growth factor therapies.

The day before endarterectomy, blood samples were obtained by peripheral venipuncture from these patients at fasting state to collect serum and plasma and to assess blood parameters. Medications reported in Table 1 were not modified in the 2 months prior to enrolment. Within the cohort, patients under treatment with a statin $(n=26)$ were compared with patients without statins $(n=12)$ for both systemic and intraplaque inflammatory and vascular parameters. Different statins and doses taken by patients were shown in Table 2. The Medical Ethics Committee of San Martino Hospital approved the study, and participants provided written informed consent. The study was conducted in compliance with the Declaration of Helsinki.

2.2. Power Estimation. Power calculation was based on prior published literature $[16,17]$. Our sample size (26 patients under statins versus 12 patients without statin) allowed us to detect large effect sizes $(>0.80)$ for systemic inflammatory markers or intraplaque parameters between symptomatic and asymptomatic patients with a power of $80 \%$, taking a twosided type I error of $5 \%$.

2.3. Systemic Inflammatory Marker Detection. Serum Creactive protein (CRP), osteopontin (OPN), osteoprotegerin (OPG), myeloperoxidase (MPO), matrix metalloproteinase (MMP)-8, MMP-9, tissue inhibitor of metalloproteinase (TIMP)-1, TIMP-2, TIMP-3, TIMP-4, and MMP9/TIMP1 levels were measured by colorimetric enzyme-linked immunosorbent assay (ELISA, from R\&D Systems, Minneapolis, Minnesota), following manufacturer's instructions. Serum RANKL levels were measured by ELISA (BioVendor $\mathrm{GmbH}$, Heidelberg, Germany), following manufacturer's instructions. Serum neutrophil elastase levels were assessed by ELISA (Bender Med Systems GmbH, Vienna, Austria), following manufacturer's instructions. The limits of detection were $0.78 \mathrm{ng} / \mathrm{mL}$ for CRP, $0.312 \mathrm{ng} / \mathrm{mL}$ for OPN, $0.0625 \mathrm{ng} / \mathrm{mL}$ for OPG, $0.156 \mathrm{ng} / \mathrm{mL}$ for MPO, $0.156 \mathrm{ng} / \mathrm{mL}$ for MMP-8, $0.312 \mathrm{ng} / \mathrm{mL}$ for MMP-9, $0.156 \mathrm{ng} / \mathrm{mL}$ for TIMP1, $0.156 \mathrm{ng} / \mathrm{mL}$ for TIMP-2, $0.0625 \mathrm{ng} / \mathrm{mL}$ for TIMP-3, $0.0781 \mathrm{ng} / \mathrm{mL}$ for TIMP-4, $0.0469 \mathrm{ng} / \mathrm{mL}$ for MMP/TIMP$1,31.25 \mathrm{pg} / \mathrm{mL}$ for RANKL, and $0.156 \mathrm{ng} / \mathrm{mL}$ for neutrophil elastase. Mean intra- and interassay coefficients of variation (CV) were below $8 \%$ for all markers. Glucose, triglycerides, total cholesterol, low-density lipoprotein (LDL) cholesterol, and high-density lipoprotein (HDL) cholesterol were routinely measured and expressed in $\mathrm{mg} / \mathrm{dL}$. Serum insulin and $\mathrm{C}$-peptide were routinely measured and expressed in $\mathrm{mU} / \mathrm{L}$ and $\mu \mathrm{g} / \mathrm{L}$, respectively.

2.4. Pro-MMP-9 Zymographic Assay. Pro-MMP-9 zymographic activity was assessed in human serum, as previously described [18]. 9\% of SDS-polyacrylamide gels were copolymerized with gelatin (Sigma, St. Louis, MO). Equal amounts of patient serum $\left(2 \mu \mathrm{L}\right.$, stored at $\left.-80^{\circ} \mathrm{C}\right)$ and $1 \mathrm{ng}$ of recombinant pro-MMP-9 standard (Calbiochem, Lucerne, Switzerland) were loaded on gels in the absence of reducing 
TABLE 1: Clinical characteristics and medications of the study population.

\begin{tabular}{|c|c|c|c|}
\hline Characteristics & Statin $(n=26)$ & No statin $(n=12)$ & $P$ value \\
\hline Age, yr (IQR) & $72.0(66.0-77.3)$ & $71.5(67.3-76.7)$ & 0.9249 \\
\hline Males, $n(\%)$ & $14(53.8)$ & $7(58.3)$ & 1.0000 \\
\hline \multicolumn{4}{|l|}{$\mathrm{CV}$ risk factors } \\
\hline \multicolumn{4}{|l|}{ Blood pressure, $\mathrm{mmHg}$} \\
\hline Systolic (IQR) & $130.0(125.0-141.3)$ & $130.0(125.0-140.0)$ & 0.6826 \\
\hline Diastolic (IQR) & $85.0(80.0-90.0)$ & $80.5(80.0-84.5)$ & 0.4686 \\
\hline Waist Circumference, cm (IQR) & $91.0(88.0-98.0)$ & $91.5(90.0-95.0)$ & 0.7414 \\
\hline Current smoking, $n(\%)$ & $7(26.9)$ & $3(25.0)$ & 1.0000 \\
\hline Type 2 diabetes, $n(\%)$ & $11(42.3)$ & $3(25.0)$ & 0.4722 \\
\hline Dislipidemia, $n(\%)$ & $16(61.5)$ & $4(33.3)$ & 0.1643 \\
\hline Hypertension, $n(\%)$ & $21(80.8)$ & $10(83.3)$ & 1.0000 \\
\hline Chronic CAD* ${ }^{*}, n(\%)$ & $7(26.9)$ & $3(25)$ & 1.0000 \\
\hline Carotid stenosis \% lumen (IQR) & $75(70-80)$ & $80(70-85)$ & 0.2349 \\
\hline \multicolumn{4}{|l|}{ Biological parameters } \\
\hline Total $\mathrm{WBC}^{\dagger}$, number $\times 10^{9} / \mathrm{L}$ (IQR) & $6.35(5.53-7.43)$ & $6.56(5.60-8.98)$ & 0.4053 \\
\hline Neutrophils, number $\times 10^{9} / \mathrm{L}$ (IQR) & $3.90(3.38-5.05)$ & $4.36(3.57-6.14)$ & 0.4996 \\
\hline Lymphocytes, number $\times 10^{9} / \mathrm{L}$ (IQR) & $1.58(1.24-1.96)$ & $1.56(1.12-1.84)$ & 0.6716 \\
\hline Monocytes, number $\times 10^{9} / \mathrm{L}$ (IQR) & $0.37(0.30-0.49)$ & $0.44(0.38-0.53)$ & 0.0429 \\
\hline Red blood cells, number $\times 10^{12} / \mathrm{L}$ (IQR) & $4.60(4.10-4.85)$ & $4.65(4.53-5.00)$ & 0.2513 \\
\hline Platelet, number ×109 /L (IQR) & $207.5(178.8-238.8)$ & $188.5(175.5-219.8)$ & 0.5096 \\
\hline Plasma fibrinogen, g/L (IQR) & $3.65(3.25-4.79)$ & $4.09(3.69-5.11)$ & 0.3146 \\
\hline Serum total-c $\mathrm{c}^{\ddagger}, \mathrm{mg} / \mathrm{dl}(\mathrm{IQR})$ & $189.0(164.0-215.0)$ & $202.0(159.3-209.3)$ & 0.6150 \\
\hline Serum LDL-c ${ }^{\mathfrak{s}}, \mathrm{mg} / \mathrm{dl}(\mathrm{IQR})$ & $104.0(81.2-130.0)$ & $118.8(77.0-132.2)$ & 0.6380 \\
\hline Serum HDL-c" & $60.0(41.5-69.0)$ & $49.5(41.5-64.0)$ & 0.4754 \\
\hline Serum triglycerides, mg/dl (IQR) & $124.0(83.5-160.0)$ & $85.0(68.0-118.8)$ & 0.2237 \\
\hline Serum glycaemia, mg/dl (IQR) & $101.0(96.5-153.0)$ & $111.0(95.8-124.0)$ & 0.6497 \\
\hline Serum insulinemia, mU/L (IQR) & $7.5(5.8-16.3)$ & $5.6(3.7-12.2)$ & 0.0747 \\
\hline Serum C-peptidemia, $\mu \mathrm{g} / \mathrm{L}$ (IQR) & $2.35(1.92-3.97)$ & $2.06(1.60-2.61)$ & 0.1894 \\
\hline Antiplatelets, $n(\%)$ & $23(88.5)$ & $10(83.3)$ & 0.6426 \\
\hline Diuretics, $n(\%)$ & $2(7.7)$ & $4(33.3)$ & 0.0661 \\
\hline $\mathrm{ACE}^{\#}$ inhibitors, $n(\%)$ & $2(7.7)$ & $1(8.3)$ & 0.2295 \\
\hline $\mathrm{ARBs}^{* *}, n(\%)$ & $11(42.3)$ & $6(50.0)$ & 0.7342 \\
\hline Beta-blockers, $n(\%)$ & $10(38.5)$ & $3(25.0)$ & 0.4859 \\
\hline Calcium channel blockers, $n(\%)$ & $9(34.6)$ & $4(33.3)$ & 1.0000 \\
\hline Oral antidiabetics, $n(\%)$ & $7(26.9)$ & $3(25.0)$ & 1.0000 \\
\hline
\end{tabular}

Continuous variables are expressed as median (interquartile range (IQR)).

${ }^{*} \mathrm{CAD}$ : coronary artery disease.

${ }^{\dagger}$ WBC: white blood cells.

* total-c: total cholesterol.

${ }^{\S}$ LDL-c: low-density lipoprotein cholesterol.

"HDL-c: high-density lipoprotein cholesterol.

${ }^{\#}$ ACE: angiotensin converting enzyme.

**ARBs: angiotensin receptor blockers.

agents. Then, gels were rinsed and stained with Coomassie Blue R-250. Zymographic results were expressed as proMMP-9 proteolytic activity and calculated on the basis of the following formula: Serum pro-MMP-9 $=\left(I_{\text {obs }} / I_{\text {std }}\right) \times W_{\text {std }}$, where $I_{\text {obs }}$ and $I_{\text {std }}$ are intensities of lytic areas produced in gels by samples and by standard pro-MMP- 9 and $W_{\text {std }}$ is the weight $(1 \mathrm{ng})$ of standard pro-MMP-9 loaded onto the gel. Zymographic data were expressed as $\mathrm{ng} / \mathrm{mL}$ of serum.
Gelatinolytic bands were measured with a gel analysis system (GeneGenius, Syngene, Cambridge, UK).

2.5. Human Carotid Plaque Specimen Processing. Shortly after surgical excision, the internal carotid plaque specimens were taken from all patients and immediately transferred at $4^{\circ} \mathrm{C}$ to the laboratory for processing. Carotid plaques had the morphology of calcified advanced lesions with a lipid-rich 
TABLE 2: Different statin treatments.

\begin{tabular}{lc}
\hline Statin (dose) & $n(\%)$ \\
\hline Rosuvastatin (5 mg/day) & $12(46.15)$ \\
Simvastatin (40 mg/day) & $6(23.08)$ \\
Atorvastatin (20 mg/day) & $8(30.77)$ \\
\hline
\end{tabular}

acellular core. The internal carotid plaque specimens were cut perpendicularly to the long axis through the point of maximum stenosis to obtain two portions (upstream and downstream blood flow) [18]. Each portion was further divided perpendicularly to the long axis in the middle into two subsegments. One half was snap-frozen in liquid nitrogen and stored at $-80^{\circ} \mathrm{C}$ for mRNA isolation, and the other half was frozen in cryoembedding medium for histological analysis.

2.6. Immunohistochemistry. Frozen upstream and downstream human carotid specimens were serially cut into eight $7 \mu \mathrm{m}$ sections per each portion separated by $105 \mu \mathrm{m}$ from each other [18]. Sections were fixed in acetone and immunostained with specific antibodies, anti-human smooth muscle actin (dilution: 1:100; Dako Corporation, Glostrup, Denmark), anti-human CD68 (dilution: 1:100; Dako Corporation), anti-human CD66b (dilution: 1:50; Beckman Coulter, Nyon, CH), anti-human matrix metalloproteinase (MMP)9 (dilution: 1:250; Sothern Biotech, Birmingham, AL), antihuman OPG (dilution: 1:20; R\&D Systems), and anti-human RANKL (dilution: 1:20; R\&D Systems). Quantifications were performed with MetaMorph software. Results for these parameters were calculated and expressed as percentages of stained area on total lesion area or number of infiltrating cells on $\mathrm{mm}^{2}$ of lesion area.

2.7. Oil Red O Staining for Lipid Content. Eight sections per portion (upstream and downstream blood flow) of human carotid plaques were stained with Oil Red $\mathrm{O}$, as previously described [18]. Sections were counterstained with Mayer's hemalum and rinsed in distilled water. Quantifications were performed with MetaMorph software. Data were calculated as ratios of stained area on total lesion area.

2.8. Sirius Red Staining for Collagen Content. Eight sections per portion (upstream and downstream blood flow) of human carotid plaques were rinsed with water and incubated with $0.1 \%$ Sirius red (Sigma Chemical Co, St Louis, MO) in saturated picric acid for $90 \mathrm{~min}$. Sections were rinsed twice with $0.01 \mathrm{~N} \mathrm{HCl}$ for $1 \mathrm{~min}$ and then immersed in water. After dehydration with ethanol for 30 seconds and cover-slipping, the sections were photographed with identical exposure settings under ordinary polychromatic or polarized light microscopy. Total collagen content was evaluated under polychromatic light. Interstitial collagen subtypes were evaluated using polarized light illumination; under this condition thicker type I collagen fibers appeared orange or red, whereas thinner type III collagen fibers were yellow or green [16]. Quantifications were performed with MetaMorph software.
Data were calculated as percentages of stained area on total lesion area.

2.9. Real-Time RT-PCR. Total mRNA was isolated with Tri Reagent (MRC Inc.) from upstream or downstream specimens of human carotid plaques. Reverse transcription was performed using the ImProm-II Reverse Transcription System (Promega, Madison, WI) according to the manufacturer's instructions. Real-time PCR (StepOnePlus, Applied Biosystems) was performed with the ABsolute QPCR Mix (ABgene).

Specific primers and probes (Table 3) were used to determine the mRNA expression of RANK, RANKL, OPG, and RPS13 (housekeeping gene). The fold change of mRNA levels was calculated by the comparative $C_{t}$ method. The measured $C_{t}$ values were first normalized to the RPS13 internal control, by calculating delta $C_{t}\left(\Delta C_{t}\right)$. This was achieved by subtracting the RPS13 $C_{t}$ values from the gene of interest $C_{t}$ value. Delta delta $C_{t}\left(\Delta \Delta C_{t}\right)$ was calculated by subtracting the designated baseline control group $\Delta C_{t}$ value from the study group $\Delta C_{t}$ values. The $\Delta \Delta C_{t}$ was then plotted as a relative fold change with the following formula: $2^{-\Delta \Delta C_{t}}$.

2.10. Statistical Analysis. Patient characteristics were described one day before endarterectomy. Patients asymptomatic for ischemic stroke and on statin treatment were compared to patients without statins using the Pearson's chi-square test or the Fisher's exact test (when appropriate) for the comparison of qualitative variables. The MannWhitney nonparametric test was used for comparisons of continuous variables. Results were expressed as medians (interquartile range (IQR)). Spearman's rank correlation coefficients were used to assess correlations between serum RANKL/OPG ratio and levels of serum neutrophil products and intraplaque parameters in both upstream and downstream regions of carotid atherosclerotic plaques, respectively. Values of $P<0.05$ (two-tailed) were considered significant. All analyses were done with GraphPad Instat software version 3.05 (GraphPad Software).

\section{Results}

3.1. Baseline Population Characteristics. Clinical features, laboratory parameters, and medications of our cohort of asymptomatic patients with severe carotid stenosis $(n=38)$ are shown in Table 1 . There was no significant difference between patients under or without treatment with a statin in terms of age, sex, classical cardiovascular risk factors, hematological parameters, and medications except for a slight but significant $(P=0.0429)$ decrease on circulating monocyte count in statin-treated group. Table 2 summarizes the statins used by the patients and their dosage. Only treatments with low doses of statins [19] were investigated in the present study (Table 2).

3.2. Statin Treatment Is Associated with the Modulation of Serum Levels of Calcification Biomarkers and MMP-9. The first goal of this study was to determine if statin treatment 
TABLE 3: Human primers used for real-time PCR.

\begin{tabular}{|c|c|c|c|c|}
\hline Gene & Function & Nucleotide sequence & Size (bp) & Accession number \\
\hline \multirow{3}{*}{ RPS13 } & Fw & $5^{\prime}$-CGTCCCCACTTGGTTGAAG-3' & \multirow{3}{*}{90} & \multirow{3}{*}{ NM_001017 } \\
\hline & $\mathrm{Rv}$ & $5^{\prime}$-CCGATCTGTGAAGGAGTAAGG-3' & & \\
\hline & Probe & 5'-ACATCTGACGACGTGAAGGAGCAGATT-3' & & \\
\hline \multirow{3}{*}{ TNFSF11 (RANKL) } & Fw & $5^{\prime}$-AAGGAGCTGTGCAAAAGGAA-3' & \multirow{3}{*}{75} & \multirow{3}{*}{ NM_003701 } \\
\hline & $\mathrm{Rv}$ & $5^{\prime}$-CATCCACCATCGCTTTCTCT-3' & & \\
\hline & Probe & $5^{\prime}$-CGTTGGATCACAGCACATCAGAGC- $3^{\prime}$ & & \\
\hline \multirow{3}{*}{ TNFRSF11A (RANK) } & Fw & $5^{\prime}$-CAGCTAATTTGTGGCACTGG-3' & \multirow{3}{*}{68} & \multirow{3}{*}{ NM_003839 } \\
\hline & $\mathrm{Rv}$ & $5^{\prime}$-ACCTGAGGACTCCTTATCTCCA-3' & & \\
\hline & Probe & $5^{\prime}$-CAATGAGGCTTGTGGCCGCCTA-3' & & \\
\hline \multirow{3}{*}{ TNFRSF11B (OPG) } & Fw & $5^{\prime}$-TGGAATAGATGTTACCCTGTGTG-3' & \multirow{3}{*}{149} & \multirow{3}{*}{ NM_002546 } \\
\hline & $\mathrm{Rv}$ & 5'-TGTGTTGCCGTTTTATCCTCT-3' & & \\
\hline & Probe & 5'-AGGCATTCTTCAGGTTTGCTGTTCC-3' & & \\
\hline
\end{tabular}

TABLE 4: Serum cardiovascular risk markers.

\begin{tabular}{lccc}
\hline Characteristics & Statin $(n=26)$ & No statin $(n=12)$ & $P$-value \\
\hline CRP $^{*}, \mathrm{mg} / \mathrm{L}$ & $2.4(0.96-4.03)$ & $4.1(1.43-8.41)$ & 0.1782 \\
OPN, ng/mL & $17.5(12.8-23.8)$ & $29.4(23.2-34.6)$ & 0.0131 \\
RANKL, pg/mL & $1022(247-1669)$ & $1839(1551-2803)$ & $62.3(62.3-432.0)$ \\
OPG, pg/mL & $167.8(62.3-318.1)$ & $27.2(15.5-43.3)$ & 0.0050 \\
RANKL/OPG ratio & $5.8(1.5-17.1)$ & $466.0(313.7-841.9)$ & 0.0182 \\
MPO, ng/mL & $265.2(101.8-531.6)$ & $325.3(143.1-450.2)$ & 0.0004 \\
Neutrophil elastase, ng/mL & $211.1(109.1-318.6)$ & $12.7(4.0-24.7)$ & 0.1014 \\
MMP ${ }^{\dagger}-8$, ng/mL & $16.5(5.8-23.7)$ & $479.0(352.8-812.5)$ \\
MMP-9, ng/mL & $231.5(134.9-431.6)$ & $13.1(11.9-18.2)$ & $14.4(2.8-23.0)$ \\
Pro-MMP-9 activity, ng/mL & $6.8(3.1-14.4)$ & $211.0(147.6-264.6)$ \\
MMP9/TIMP-1, ng/mL & $10.6(6.4-18.4)$ & $97.0(73.0-112.2)$ \\
TIMP ${ }^{\ddagger}-1$, ng/mL & $191.3(120.0-216.7)$ & $12.5(6.2-13.8)$ & 0.7579 \\
TIMP-2, ng/mL & $101.4(65.4-124.5)$ & $4.2(4.0-6.1)$ & 0.0461 \\
TIMP-3, ng/mL & $8.1(4.7-14.9)$ & 0.8839 \\
TIMP-4, ng/mL & $5.4(3.6-6.6)$ & 0.3389 \\
\hline
\end{tabular}

Data are expressed as median (interquartile range (IQR)).

${ }^{*}$ CRP: C-reactive protein.

${ }^{\dagger}$ MMP: matrix metalloproteinase.

${ }^{\ddagger}$ TIMP: tissue inhibitor of metalloproteinase.

was associated with improvements in the systemic levels of cardiovascular risk factors, calcification biomarkers, and neutrophil degranulation products, with CRP being the first biomarker to be analyzed. Statin-treated patients had similar CRP serum levels as compared to statin-untreated controls (Table 4). With respect to calcification biomarkers, serum levels of OPN and RANKL were significantly reduced, while OPG was increased in statin-treated patients as compared to patients without statins. In line with the observed changes in RANKL and OPG levels, the RANKL/OPG ratio (indicating the circulating RANKL free fraction) was significantly decreased in statin-treated patients as compared to controls. With respect to neutrophil degranulation products, we did not find any difference between the two patient groups in terms of serum MPO, neutrophil elastase, and MMP-8 levels and of protective tissue inhibitor of metalloproteinases
(TIMP-1, -2, -3, and -4) (Table 4). However, the MMP-9 level and pro-MMP-9 activity were significantly reduced in patients receiving statin therapy as compared to those without statins (Table 4).

3.3. Statin Treatment Is Associated with Reduction in Neutrophil Infiltrates and Vulnerability in Carotid Plaque Portions Downstream Blood Flow. Taking into account the elevated heterogeneity of human carotid plaques $[17,18,20]$, the effect of statin treatment on intraplaque inflammatory patterns and calcification biomarkers was assessed in different regions (upstream and downstream blood flow, resp.). In upstream portions, no differences were found between the two patient groups in terms of lipid and collagen content and of cell composition (SMCs, macrophages, neutrophils, and MMP-9) (Table 5, Figures 1 and 2). In downstream regions, 
TABLE 5: Parameters of intraplaque vulnerability.

\begin{tabular}{|c|c|c|c|}
\hline Characteristics & Statin $(n=26)$ & No statin $(n=12)$ & $P$-value \\
\hline \multicolumn{4}{|l|}{ Upstream portion } \\
\hline$\%$ of lipid & $4.63(1.84-8.63)$ & $4.36(3.17-5.54)$ & 0.6524 \\
\hline$\%$ of total collagen & $41.24(18.91-52.70)$ & $30.43(21.25-43.09)$ & 0.6786 \\
\hline$\%$ of collagen I & $8.97(4.27-15.98)$ & $6.42(4.71-10.04)$ & 0.4385 \\
\hline$\%$ of collagen III & $21.79(15.32-37.75)$ & $20.33(16.67-31.94)$ & 0.9569 \\
\hline$\%$ of smooth muscle cell-rich area & $8.85(3.75-19.57)$ & $8.00(3.22-14.48)$ & 0.6013 \\
\hline$\%$ of macrophage-rich area & $2.96(0.88-9.06)$ & $2.08(0.27-6.69)$ & 0.4823 \\
\hline Neutrophils $/ \mathrm{mm}^{2}$ & $0.75(0.52-1.32)$ & $0.81(0.30-12.47)$ & 0.6786 \\
\hline$\%$ of $\mathrm{MMP}^{*}-9$ & $0.71(0.33-1.36)$ & $0.39(0.21-7.19)$ & 0.6524 \\
\hline $\mathrm{RANK}^{\dagger} \mathrm{mRNA}$, fold increase & $0.49(0.33-1.15)$ & $0.81(0.31-1.74)$ & 0.5725 \\
\hline $\mathrm{RANKL}^{*} \mathrm{mRNA}$, fold increase & $0.78(0.30-1.24)$ & $0.61(0.32-0.98)$ & 0.8859 \\
\hline $\mathrm{OPG}^{\S} \mathrm{mRNA}$, fold increase & $0.84(0.57-1.47)$ & $0.83(0.50-1.17)$ & 0.7944 \\
\hline$\%$ of OPG & $2.71(1.77-6.06)$ & $0.50(0.10-2.34)$ & 0.0150 \\
\hline \multicolumn{4}{|l|}{ Downstream portion } \\
\hline$\%$ of lipid & $4.39(2.83-8.69)$ & $4.94(2.13-6.63)$ & 0.4884 \\
\hline$\%$ of total collagen & $18.45(2.07-45.87)$ & $5.06(0.50-10.28)$ & 0.0289 \\
\hline$\%$ of collagen I & $5.56(0.67-20.74)$ & $0.67(0.11-3.37)$ & 0.0345 \\
\hline$\%$ of collagen III & $10.61(2.15-25.11)$ & $1.98(0.70-8.57)$ & 0.0728 \\
\hline$\%$ of smooth muscle cell-rich area & $3.87(1.50-5.15)$ & $3.59(2.42-4.30)$ & 0.8173 \\
\hline$\%$ of macrophage-rich area & $1.97(1.02-8.29)$ & $9.00(2.24-11.70)$ & 0.1060 \\
\hline Neutrophils $/ \mathrm{mm}^{2}$ & $0.26(0.15-0.93)$ & $3.77(1.20-8.16)$ & 0.0001 \\
\hline$\%$ of MMP-9 & $4.05(0.68-12.30)$ & $10.12(3.86-13.81)$ & 0.0574 \\
\hline RANK mRNA, fold increase & $0.99(0.48-2.77)$ & $0.62(0.38-2.43)$ & 0.4601 \\
\hline RANKL mRNA, fold increase & $0.84(0.45-1.25)$ & $0.59(0.17-1.60)$ & 0.5165 \\
\hline OPG mRNA, fold increase & $1.38(1.06-1.71)$ & $1.06(0.80-1.60)$ & 0.2117 \\
\hline$\%$ of OPG & $0.27(0.01-0.96)$ & $0.28(0.02-0.67)$ & 0.7746 \\
\hline
\end{tabular}

Data are expressed as median (interquartile range (IQR)).

*MMP: matrix metalloproteinase.

${ }^{\dagger}$ RANK: receptor activator of nuclear factor- $\kappa$ B.

${ }^{\ddagger}$ RANKL: receptor activator of nuclear factor- $\kappa \mathrm{B}$ ligand.

${ }^{\S}$ OPG: osteoprotegerin.

the total collagen and collagen I contents were significantly higher in the statin-treated group as compared to the group with no statin use. Notably, this effect was associated with a virtual abrogation of intraplaque neutrophil infiltration in patients treated with statins as compared to untreated patients. Despite being close to significance $(P=0.0574)$, a similar trend in intraplaque content of the neutrophilic product MMP-9 was also observed (Table 5). No difference between the two patient groups was found in terms of intraplaque lipids and of infiltration by other cell subsets, such as SMCs and macrophages (Table 5).

Calcification biomarkers have previously been reported to be expressed within atherosclerotic plaques [21]. Thus, we focused on the expression of these markers in our cohort of patients both at mRNA and protein level. RANKL was almost undetectable in both groups at both mRNA (upstream plaques: detectable in 14 statin and 10 no statin; downstream plaques: in 17 statin and 11 no statin) and protein levels (detectable in 4 downstream portions of carotid plaques from statin-treated patients, data not shown). Statin treatment was not associated with an altered expression of RANKL, RANK (RANKL's receptor), and OPG mRNA as compared to control patients without statins (Table 5). At the protein level, intraplaque OPG expression was weak in both groups (Table 5). A significant increase in OPG protein levels was shown in upstream regions of carotid plaques from statintreated versus untreated patients, whereas no statistically significant difference in downstream plaque portions could be detected (Table 5). Finally, the expression pattern of OPG in carotid plaques suggested that this protein did not colocalize with macrophages or neutrophils but rather with intimal SMCs and lipids (Figures 1 and 2). This result was similar in both patients' groups (Figures 1 and 2).

3.4. RANKL/OPG Ratio Positively Correlates with Neutrophil Degranulation Products Both in the Systemic Circulation and within Carotid Plaques. Spearman rank correlations were performed in order to identify potential associations between free serum RANKL levels (RANKL/OPG ratio) on the one 

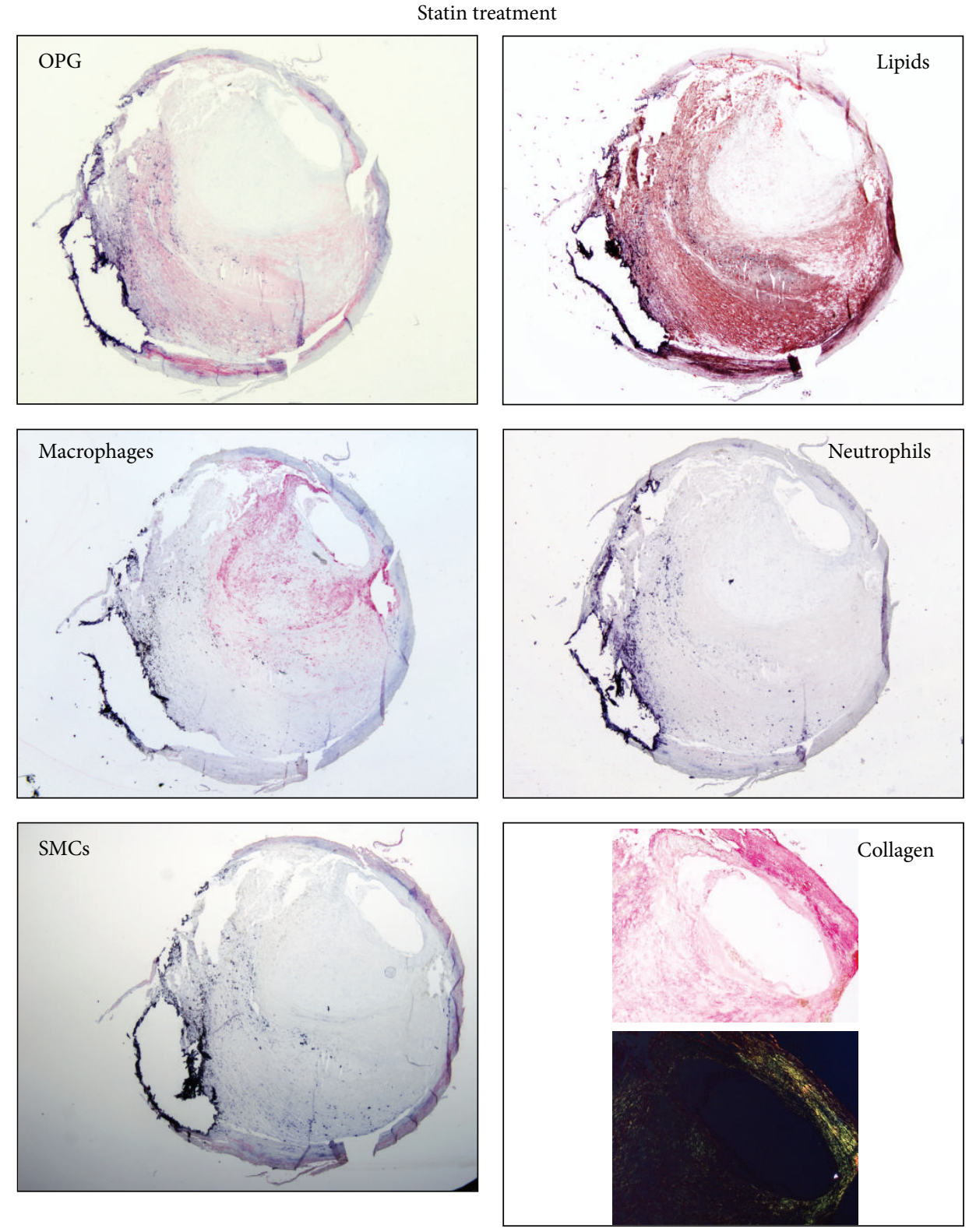

FIGURE 1: Representative microphotographs of consecutive cryosections from upstream regions of carotid plaques from patients on statin treatment. Staining for osteoprotegerin (OPG), lipids, macrophages, neutrophils and smooth muscle cells (SMCs), and collagen is shown.

hand and systemic and intraplaque neutrophil products on the other in our two groups of patients (statin treatment versus no statin treatment). RANKL/OPG ratio was found to positively correlate with serum CRP, MPO, and neutrophil elastase levels, as well as with serum pro-MMP-9 activity (Table 6). Though not statistically significant $(P=0.0614)$, a positive association between RANKL/OPG ratio and MMP9 serum levels was observed (Table 6). No significant association between RANKL/OPG ratio and serum MMP-8 was detected (Table 6). Serum RANKL/OPG ratio was also shown to inversely correlate with intraplaque lipid content in upstream regions of carotid plaques and to positively correlate with neutrophil and MMP-9 content in downstream portions. No additional significant correlations were shown for other intraplaque parameters, such as collagen content, SMCs, and macrophages, in the different intraplaque regions (Table 6).

\section{Discussion}

The main result of this study is represented by the finding that an ongoing treatment with statins is associated with alterations in serum levels of biomarkers of plaque calcification such as OPN, RANKL, and OPG in asymptomatic patients with a severe carotid stenosis undergoing a carotid endarterectomy. In particular, the free circulating fraction of RANKL (not bound to OPG, expressed as RANKL/OPG ratio) was strongly reduced in statin-treated patients as 


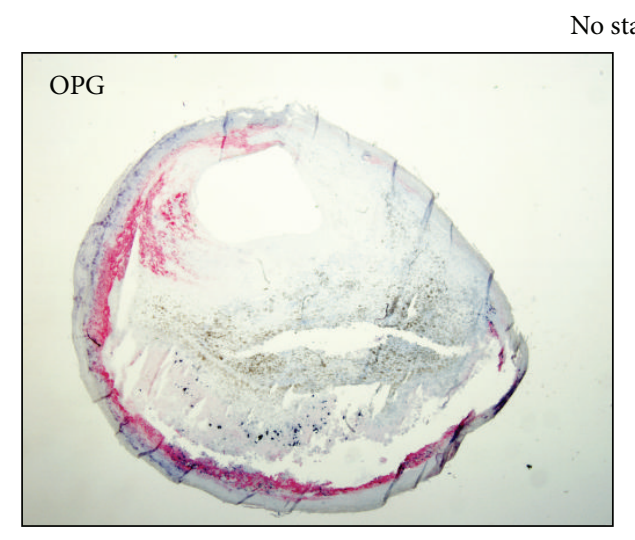

\section{o statins}
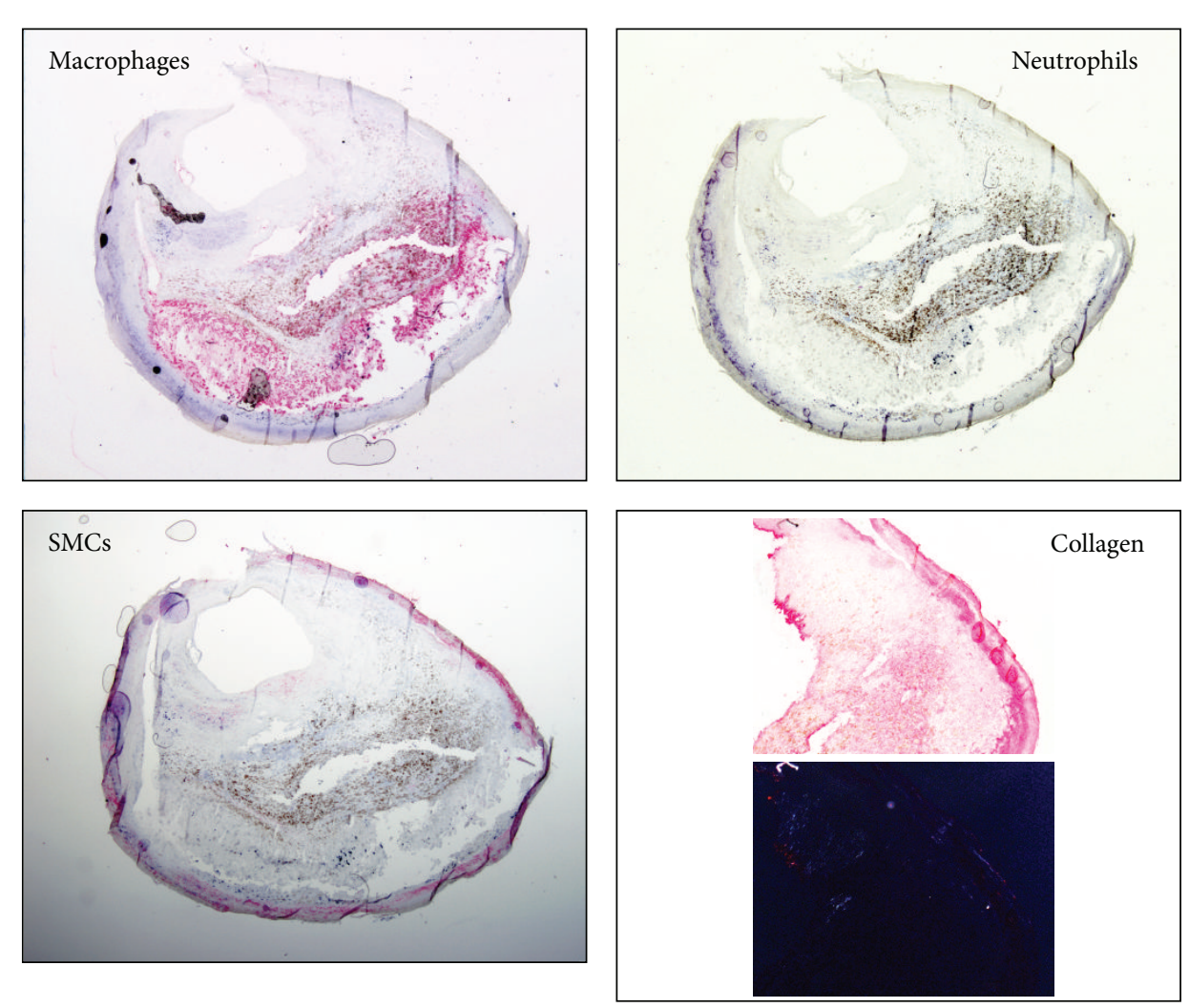

FIGURE 2: Representative microphotographs of consecutive cryosections from upstream regions of carotid plaques from patients without statins. Staining for osteoprotegerin (OPG), lipids, macrophages, neutrophils and smooth muscle cells (SMCs), and collagen is shown.

compared to controls without a statin. Conversely, we did not detect any relevant change in intraplaque RANKL/OPG levels between statin-treated and -untreated patients. Notably, we were only able to detect very low intraplaque RANKL levels, suggesting that this mediator may be preferentially expressed in the blood stream, instead of within atherosclerotic plaques [9]. Our data also suggest a new, potential, beneficial effect of statins as they may directly reduce the levels of circulating free RANKL, thereby preventing its detrimental effects on proatherosclerotic cell subsets (including neutrophils). A potential reduction in both OPG and RANKL serum levels after atorvastatin treatment was previously shown by Dimitrow and coworkers in patients with aortic sclerosis or mild aortic stenosis [22]. In this paper, we confirmed that statin treatment was associated with a reduction in RANKL serum levels, but, surprisingly, we showed an increase in serum OPG (the decoy receptor of soluble RANKL that blocks its bioactivity). The role of OPG in cardiovascular diseases requires further clarifications, since its levels have been associated with cardiovascular risk without reporting a clear proatherosclerotic direct bioactivity of this molecule [23-25]. In $\mathrm{OPG}^{-1-}$ mice, treatment with recombinant OPG induced signs of fibrosis, promoted intraplaque SMCs accumulation, collagen fiber formation, and development of fibrous caps, thus supporting a pathogenic role of OPG in the development, progression, and instability of atherosclerotic lesions 
TABLE 6: Spearman rank correlation between serum RANKL/OPG ratio and systemic and intraplaque parameters (upstream or downstream regions) of vulnerability.

\begin{tabular}{lcc}
\hline & $\begin{array}{c}\text { Spearman's correlation } \\
\text { coefficient }(r)\end{array}$ & P-value \\
\hline Serum RANKL/OPG ratio & & \\
Systemic & & \\
CRP, mg/L & 0.3549 & 0.0311 \\
MPO, ng/mL & 0.6036 & 0.0001 \\
Neutrophil elastase, ng/mL & 0.5223 & 0.0009 \\
MMP-8, ng/mL & 0.0436 & 0.7977 \\
MMP-9, ng/mL & 0.3106 & 0.0614 \\
Pro-MMP-9 activity, ng/mL & 0.3248 & 0.0498 \\
Intraplaque upstream & & \\
versus lipids & -0.3456 & 0.0489 \\
versus total collagen & 0.0077 & 0.9661 \\
versus collagen I & 0.0879 & 0.6267 \\
versus collagen III & 0.0906 & 0.6162 \\
versus SMCs & -0.0832 & 0.6452 \\
versus macrophages & -0.0552 & 0.7605 \\
versus neutrophils & 0.1180 & 0.5132 \\
versus MMP-9 & -0.2647 & 0.1366 \\
Intraplaque downstream & -0.0946 & 0.5947 \\
versus lipids & -0.1383 & 0.4355 \\
versus total collagen & -0.1636 & 0.3551 \\
versus collagen I & -0.0805 & 0.6508 \\
versus collagen III & 0.0118 & 0.9473 \\
versus SMCs & 0.375610 \\
versus macrophages & & 0.1605 \\
versus neutrophils & & 0.0285 \\
versus MMP-9 & & 0.0418 \\
\hline
\end{tabular}

[26]. However, additional studies that should be aimed, for instance, at identifying OPG serum cutoff values, are needed to clarify the exact nature of OPG as an innocent bystander or an active player in cardiovascular diseases.

The lack of efficacy of statins in the modulation of intraplaque RANKL/OPG levels might be also explained by different findings. First of all, all statins were administered to the patients from our cohort at a low dose [19]. Thus, their tissue concentration may have been not high enough to reduce intraplaque inflammation. To some extent, this hypothesis is corroborated by the weak improvements in plaque stability parameters that we found in patients on statin as compared to untreated controls. Namely, we only detected a small reduction in neutrophil and MMP-9 content and an increase in intraplaque collagen in the downstream portions of carotid plaques in statin-treated patients versus controls, while Crisby and colleagues [16] had previously found that pravastatin treatment markedly improved carotid plaque stability as compared with control patients by reducing macrophages, T cells, and MMP-2 and by increasing protective intraplaque collagen content.
Another possible reason for our inability to detect a modulation of intraplaque RANKL/OPG levels is that our approach investigated atherosclerotic plaques as very heterogeneous tissues (as a result of different types of shear stress exposure) [20], while Crisby and colleagues evaluated the plaque a single homogeneous tissue. Thus, potential differences from the study may reflect the different approach.

Finally, the fact that different statins (rosuvastatin, simvastatin, and atorvastatin) were administrated to the patients in our study might also explain, at least in part, the limited activity of statins on intraplaque parameters that we observed. A similar result was also reported by Verhoeven and coworkers [27], who found that macrophage content might be particularly affected by atorvastatin treatment but not by other statins.

Notably, in out cohort of patients, treatment with statins did not reduce plaque lipid-rich core size, which is a key vulnerability marker in atherosclerotic plaques. This result may be surprising since Crisby and colleagues [16] clearly demonstrated that the plaques of pravastatin-treated patients with symptomatic carotid artery stenosis had substantially less lipid (3 times less) as compared to control subjects. On the other hand, our findings are supported by a more recent study on coronary atherosclerosis, in which the authors demonstrated that statins significantly decreased plaque size only if LDL-c was $<100 \mathrm{mg} / \mathrm{mL}$ [28]. In our study, we did not observe such important reduction in LDL-c levels in patients treated with statins (median value $104 \mathrm{mg} / \mathrm{dL}$ and LDL-c levels $<100 \mathrm{mg} / \mathrm{dL}$ in only 12 patients $(46.2 \%)$ ). Therefore, taking all of these aspects into account and in accordance with the current international recommendations [12], our study suggests that the use of high-dose and long-term lipidlowering strategy with a statin has to be considered when the goal is to reduce intraplaque vulnerability and cardiovascular adverse events in patients with advanced atherosclerosis [29, 30].

Another important result of this study is represented by the confirmation of serum direct correlations between free RANKL levels and neutrophil products. A significant positive association between levels of RANKL/OPG ratio and other neutrophil products (such as MPO and neutrophil elastase) was also demonstrated, confirming a strong induction of neutrophil degranulation mediated by this cytokine [9]. This robust activation of neutrophils (positively associated in our study with serum RANKL levels) was particularly observed in the systemic circulation instead of within the carotid plaque. Confirming a previous study showing an active role of RANKL in circulating neutrophil degranulation of proatherosclerotic products (such as MMP-9) in patients with increased CAC and in vitro [9], this study further confirmed these cells as a major source of proteinases favoring systemic atherosclerotic vulnerability.

This pilot explanatory study has several imitations. Firstly, the number of participants is relatively small (also with a disproportion between statin-treated patients $(n=26)$ and untreated controls $(n=12))$, thus preventing the detection of small differences in all markers between the two groups. Secondly, as we have mentioned in the discussion, we do not know how different lipid-lowering regimens 
(i.e., different statins and high-dose versus low-dose therapeutic approaches) can modulate the inflammatory burden within serum and atherosclerotic plaques. Anyway, our results might unveil a novel "pleiotropic" activity for statins selectively targeting RANKL/OPG system and potentially neutrophil activation and plaque calcification. In conclusion, we demonstrated that patients treated with a statin (lowdose) for severe carotid stenosis had reduced serum levels of free RANKL (RANKL/OPG ratio) as compared to controls without statins. This reduction of RANKL levels was positively associated with a concomitant reduction in neutrophil degranulation products, primarily in the systemic circulation and also within carotid plaques. This hypothesis-generating study may suggest that treatments that more selectively target the RANKL/OPG system might be beneficial in advanced atherosclerosis to reduce neutrophil-mediated patient vulnerability.

\section{Conflict of Interests}

All authors declare that no conflict of interests exists.

\section{Acknowledgments}

This research was funded by EU FP7, Grant no. 201668, AtheroRemo, to Dr. F. Mach. This work was also supported by the Swiss National Science Foundation Grants to Dr. F. Mach (no. 310030-118245) and Dr. F. Montecucco (no. 32003B134963/1). This work was also supported by a Grant from Novartis Foundation to Dr. F. Montecucco and by the Italian Ministry of Health (GR-2008-1135635) to Dr. A. Nencioni.

\section{References}

[1] J. Yeboah, J. J. Carr, J. G. Terry et al., "Computed tomographyderived cardiovascular risk markers, incident cardiovascular events, and all-cause mortality in nondiabetics: the multiethnic study of atherosclerosis," European Journal of Preventive Cardiology. In press.

[2] D. M. Hermann, J. Gronewold, N. Lehmann et al., "Coronary artery calcification is an independent stroke predictor in the general population," Stroke, vol. 44, pp. 1008-1013, 2013.

[3] W. E. Shaalan, H. Cheng, B. Gewertz et al., "Degree of carotid plaque calcification in relation to symptomatic outcome and plaque inflammation," Journal of Vascular Surgery, vol. 40, no. 2, pp. 262-269, 2004.

[4] M. Naghavi, P. Libby, E. Falk et al., "From vulnerable plaque to vulnerable patient: a call for new definitions and risk assessment strategies: part I," Circulation, vol. 108, no. 14, pp. 1664-1672, 2003.

[5] M. Naghavi, P. Libby, E. Falk et al., "From vulnerable plaque to vulnerable patient: a call for new definitions and risk assessment strategies: part II," Circulation, vol. 108, no. 15, pp. 1772-1778, 2003.

[6] E. M. Gravallese, "Osteopontin: a bridge between bone and the immune system," Journal of Clinical Investigation, vol. 112, no. 2, pp. 147-149, 2003.

[7] M. Schoppet, K. T. Preissner, and L. C. Hofbauer, "RANK ligand and osteoprotegerin: paracrine regulators of bone metabolism and vascular function," Arteriosclerosis, Thrombosis, and Vascular Biology, vol. 22, no. 4, pp. 549-553, 2002.

[8] S. A. Steitz, M. Y. Speer, M. D. McKee et al., "Osteopontin inhibits mineral deposition and promotes regression of ectopic calcification," The American Journal of Pathology, vol. 161, no. 6, pp. 2035-2046, 2002.

[9] A. Quercioli, F. Mach, M. Bertolotto et al., "Receptor activator of NF- $\kappa$ B ligand (RANKL) increases the release ofneutrophil products associated with coronary vulnerability," Thrombosis and Haemostasis, vol. 107, no. 1, pp. 124-139, 2012.

[10] D. H. Henry, L. Costa, F. Goldwasser et al., "Randomized, double-blind study of denosumab versus zoledronic acid in the treatment of bone metastases in patients with advanced cancer (excluding breast and prostate cancer) or multiple myeloma," Journal of Clinical Oncology, vol. 29, no. 9, pp. 1125-1132, 2011.

[11] A. Lipton, K. Fizazi, A. T. Stopeck et al., "Superiority of denosumab to zoledronic acid for prevention of skeletal-related events: a combined analysis of 3 pivotal, randomised, phase 3 trials," European Journal of Cancer, vol. 48, no. 16, pp. 30823092, 2012.

[12] Z. Reiner, A. L. Catapano, G. De Backer et al., "ESC/EAS guidelines for the management of dyslipidaemias: the task force for the management of dyslipidaemias of the European Society of Cardiology (ESC) and the European Atherosclerosis Society (EAS)," European Heart Journal, vol. 32, no. 14, pp. 1769-1818, 2011.

[13] F. Akdim, S. I. van Leuven, J. J. P. Kastelein, and E. S. G. Stroes, "Pleiotropic effects of statins: stabilization of the vulnerable atherosclerotic plaque?" Current Pharmaceutical Design, vol. 13, no. 10, pp. 1003-1012, 2007.

[14] B. G. Brown, X.-Q. Zhao, D. E. Sacco, and J. J. Albers, "Lipid lowering and plaque regression: new insights into prevention of plaque disruption and clinical events in coronary disease," Circulation, vol. 87, no. 6, pp. 1781-1791, 1993.

[15] P. M. Ridker, E. Danielson, F. A. H. Fonseca et al., "Rosuvastatin to prevent vascular events in men and women with elevated Creactive protein," The New England Journal of Medicine, vol. 359, no. 21, pp. 2195-2207, 2008.

[16] M. Crisby, G. Nordin-Fredriksson, P. K. Shah, J. Yano, J. Zhu, and J. Nilsson, "Pravastatin treatment increases collagen content and decreases lipid content, inflammation, metalloproteinases, and cell death in human carotid plaques: implications for plaque stabilization," Circulation, vol. 103, no. 7, pp. 926-933, 2001.

[17] F. Montecucco, V. Di Marzo, R. F. Da Silva et al., "The activation of the cannabinoid receptor type 2 reduces neutrophilic protease-mediated vulnerability in atherosclerotic plaques," European Heart Journal, vol. 33, no. 7, pp. 846-856, 2012.

[18] F. Montecucco, N. Vuilleumier, S. Pagano et al., "Antiapolipoprotein A-1 auto-antibodies are active mediators of atherosclerotic plaque vulnerability," European Heart Journal, vol. 32, no. 4, pp. 412-421, 2011.

[19] H. Naci, J. J. Brugts, R. Fleurence, and A. E. Ades, "Dosecomparative effects of different statins on serum lipid levels: a network meta-analysis of 256,827 individuals in 181 randomized controlled trials," European Journal of Preventive Cardiology, vol. 20, no. 4, pp. 658-670, 2013.

[20] F. Montecucco, S. Lenglet, A. Gayet-Ageron et al., "Systemic and intraplaque mediators of inflammation are increased in patients symptomatic for ischemic stroke," Stroke, vol. 41, no. 7, pp. 1394$1404,2010$. 
[21] C. R. Dhore, J. P. M. Cleutjens, E. Lutgens et al., "Differential expression of bone matrix regulatory proteins in human atherosclerotic plaques," Arteriosclerosis, Thrombosis, and Vascular Biology, vol. 21, no. 12, pp. 1998-2003, 2001.

[22] P. P. Dimitrow, M. Jawień, and A. Gackowski, "The influence of statins on levels of calcification biomarkers in patients with aortic sclerosis or mild aortic stenosis," The Journal of Heart Valve Disease, vol. 20, no. 1, pp. 18-22, 2011.

[23] W. S. Browner, L.-Y. Lui, and S. R. Cummings, "Associations of serum osteoprotegerin levels with diabetes, stroke, bone density, fractures, and mortality in elderly women," Journal of Clinical Endocrinology and Metabolism, vol. 86, no. 2, pp. 631637, 2001.

[24] S. Jono, Y. Ikari, A. Shioi et al., "Serum osteoprotegerin levels are associated with the presence and severity of coronary artery disease," Circulation, vol. 106, no. 10, pp. 1192-1194, 2002.

[25] M. Schoppet, A. M. Sattler, J. R. Schaefer, M. Herzum, B. Maisch, and L. Hofbauer, "Increased osteoprotegerin serum levels in men with coronary artery disease," Journal of Clinical Endocrinology and Metabolism, vol. 88, no. 3, pp. 1024-1028, 2003.

[26] N. Bucay, I. Sarosi, C. R. Dunstan et al., "Osteoprotegerindeficient mice develop early onset osteoporosis and arterial calcification," Genes and Development, vol. 12, no. 9, pp. 12601268, 1998.

[27] B. A. N. Verhoeven, F. L. Moll, J. A. F. Koekkoek et al., "Statin treatment is not associated with consistent alterations in inflammatory status of carotid atherosclerotic plaques: a retrospective study in 378 patients undergoing carotid endarterectomy," Stroke, vol. 37, no. 8, pp. 2054-2060, 2006.

[28] J. Tian, X. Gu, Y. Sun et al., "Effect of statin therapy on the progression of coronary atherosclerosis," BMC Cardiovascular Disorders, vol. 12, article 70, 2012.

[29] C. P. Cannon, B. A. Steinberg, S. A. Murphy, J. L. Mega, and E. Braunwald, "Meta-analysis of cardiovascular outcomes trials comparing intensive versus moderate statin therapy," Journal of the American College of Cardiology, vol. 48, no. 3, pp. 438-445, 2006.

[30] M. J. Tikkanen, M. Szarek, R. Fayyad et al., "Total cardiovascular disease burden: comparing intensive with moderate statin therapy: insights from the IDEAL (Incremental Decrease in End Points through Aggressive Lipid Lowering) trial," Journal of the American College of Cardiology, vol. 54, no. 25, pp. 2353-2357, 2009. 


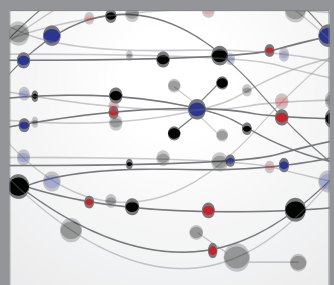

The Scientific World Journal
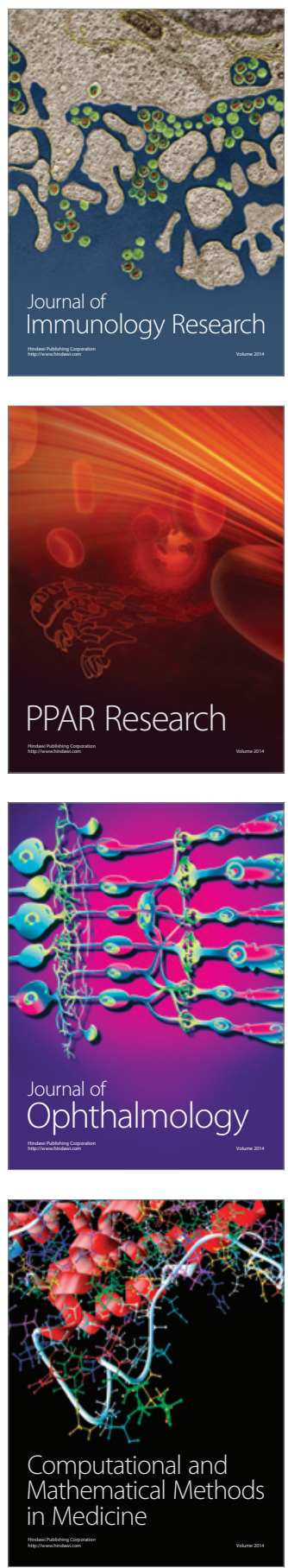

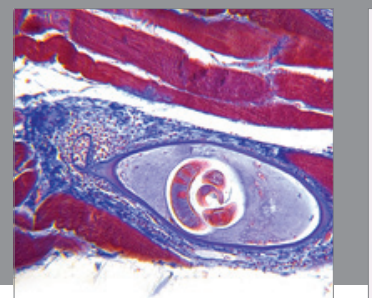

Gastroenterology

Research and Practice
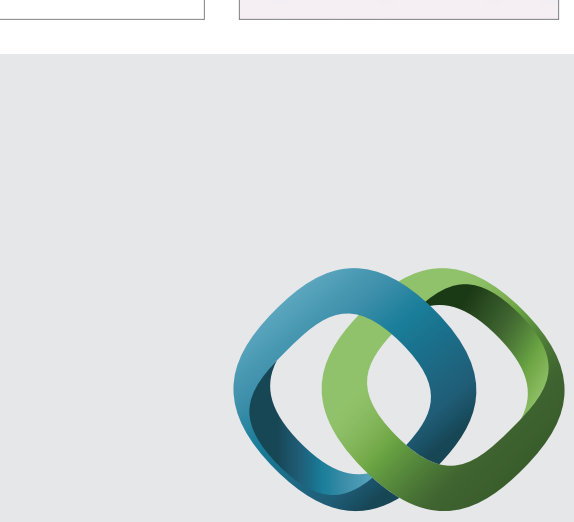

\section{Hindawi}

Submit your manuscripts at

http://www.hindawi.com
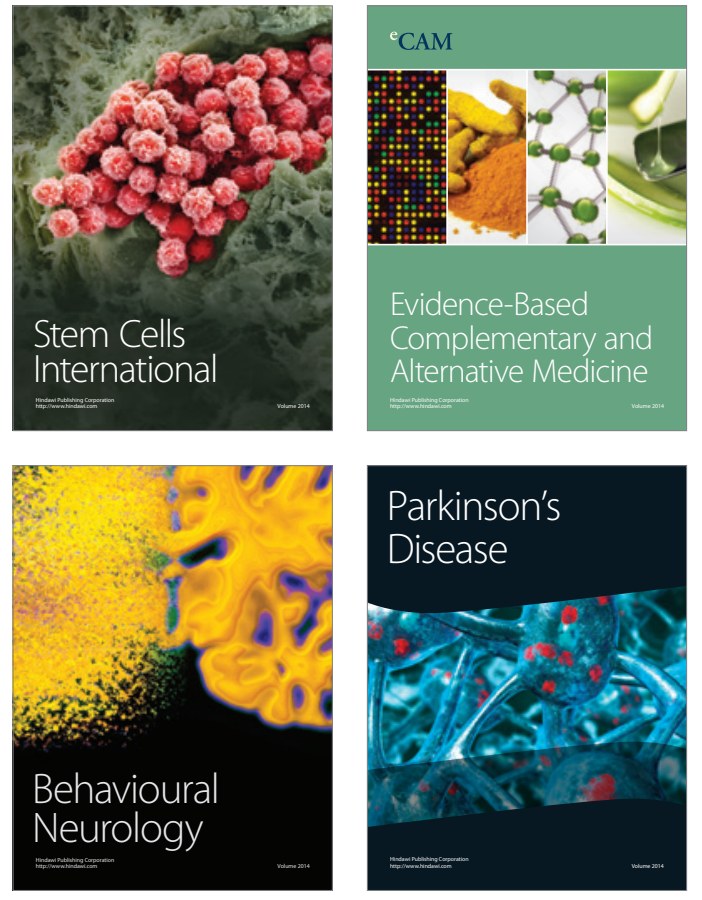
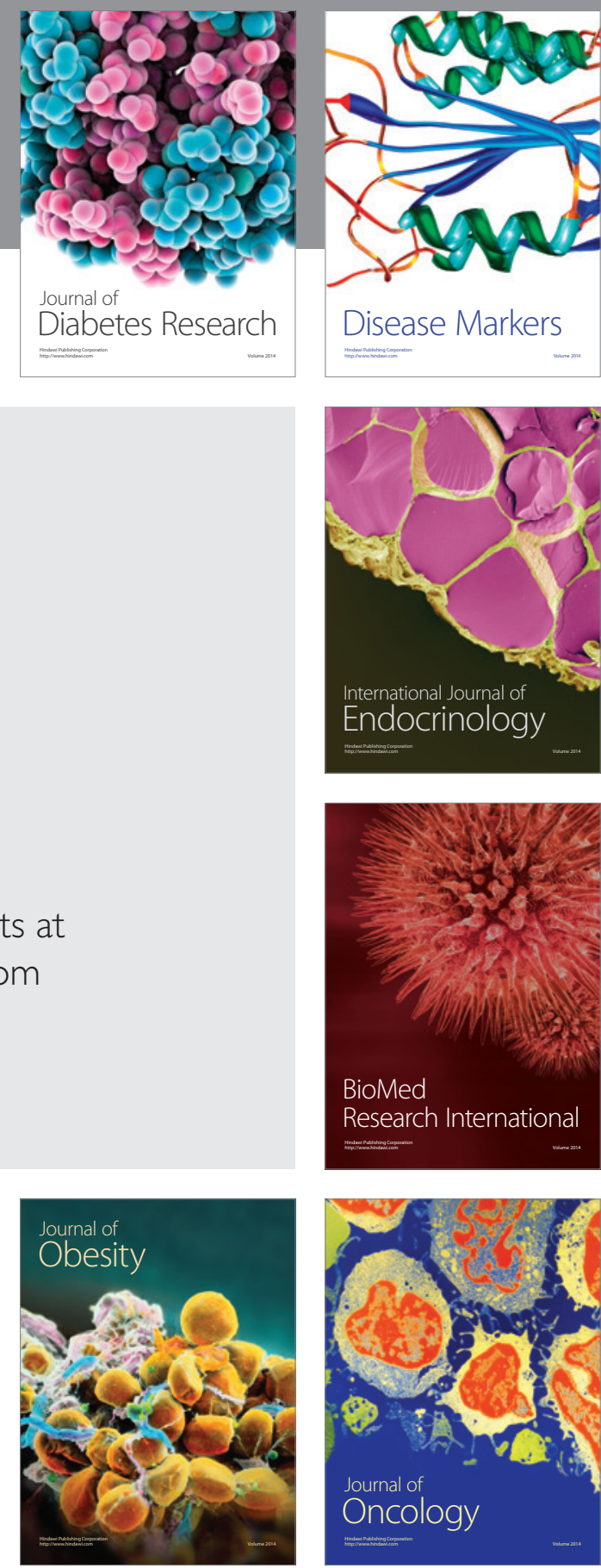

Disease Markers
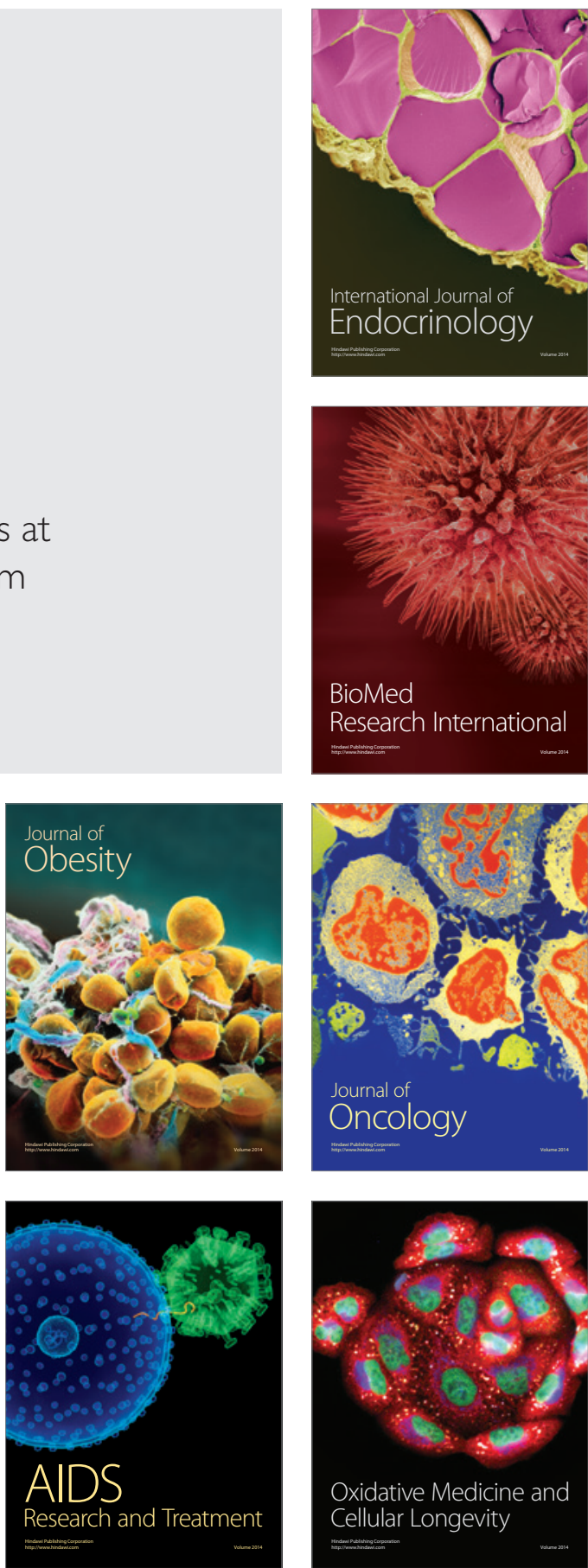